\title{
Protected percutaneous coronary intervention - a new road for success in treating complex patients
}

\author{
Fadi Al-Rashid, Heike A. Hildebrandt, Philipp Kahlert \\ Department of Cardiology, West German Heart and Vascular Center, Essen University Hospital, University Duisburg-Essen, Essen, Germany
}

Adv Interv Cardiol 2016; 12, 3 (45): 200-202

DOI: 10.5114/aic.2016.61639

\begin{abstract}
Advances in material and procedural techniques have improved the ability to perform percutaneous coronary intervention $(\mathrm{PCl})$ in challenging and complex anatomies. Yet, technical feasibility of $\mathrm{PCl}$ is not the only issue in the treatment of complex coronary artery disease, which may be approached by surgery or by $\mathrm{PCl}$. Complexity of the patient is the other, and is probably even more important. The SYNTAX trial has shown that patients with low anatomical complexity can be treated safely by $\mathrm{PCl}$, while patients with high anatomical complexity should be treated by surgery $[1,2]$. However, this does not hold true for complex patients with a high perioperative risk, who are frequently encountered in clinical practice today. Such patients present not only with complex coronary anatomies (e.g. left main stenosis, multivessel disease, last remaining vessel), but also with hemodynamic impairment (e.g. impaired left ventricular function, hemodynamic instability) and complex comorbidities (e.g. recent myocardial infarction, congestive heart failure, diabetes, chronic obstructive pulmonary disease, renal failure, valvular heart disease, increased age). Hence, decision making in general has to be carefully based on the anatomical risk (i.e. the complexity of the coronary artery disease) on one hand and on the surgical, perioperative risk on the other (Figures $1 \mathrm{~A}, \mathrm{~B}$ ).
\end{abstract}

In routine clinical practice, high-risk patients with high-risk anatomies are often judged as inoperable or at too high risk for surgery by the heart team and referred for interventional treatment by $\mathrm{PCl}$. But how can the interventionalist ensure a good procedural outcome in patients presenting with severe impairment of left ventricular function and left main disease? Of note, these were exactly the patients who were treated in the article of Dudek et al. presented in the current issues of Advances in Interventional Cardiology [3].

Current guidelines suggest that elective insertion of an appropriate hemodynamic support device as an adjunct to $\mathrm{PCl}$ may be reasonable in carefully selected high-risk patients $[4,5]$. Though somewhat general, this recommendation contains the principle of so-called protected PCls.

Coronary ischemia as a result of repeated temporary coronary occlusions (during ballon-angioplasty and stenting) can transiently worsen during $\mathrm{PCl}$, which may lead to peri- and postprocedural adverse events. This is of utmost importance in patients with left ventricular dysfunction and large areas of myocardium at risk who have little reserve. As a consequence, repeated drops in blood pressure may occur during the intervention, and operators are often in the dilemma to proceed in an "in and out as fast as possible" manner rather than to achieve an optimal result or complete revascularization. The maintenance of perfusion pressure by an appropriate hemodynamic support device promises protection from the risk of hemodynamic compromise and may thus allow complete revascularization, which should translate into a better outcome.

These assumptions are substantiated by data and subanalyses from the PROTECT II trial [6]: The PROTECT II trial was the largest prospective, randomized clinical trial on the use of percutaneous left ventricular assist devices during high-risk $\mathrm{PCl}$. Overall, 452 symptomatic patients with complex three-vessel disease or left main disease and severely impaired left ventricular function were randomized between left ventricular support by an intraaortic balloon pump (IABP) or the Impella 2.5 device $(n=$ 226). The primary endpoint was the 30-day incidence of major adverse events. However, the study was discontinued prematurely (planned enrollment: 654 patients) due to futility based on the observed conditional power of the 30-day results on an interim analysis. While the Impella 2.5 device provided superior hemodynamic support compared to the IABP, the primary endpoint was not

\section{Corresponding author:}

Philipp Kahlert, Department of Cardiology, West German Heart and Vascular Center, Essen University Hospital, University Duisburg-Essen, Hufelandstr. 55, 45122 Essen, Germany, e-mail: Philipp.Kahlert@uk-essen.de

Received: 4.07.2016, accepted: 4.07.2016. 


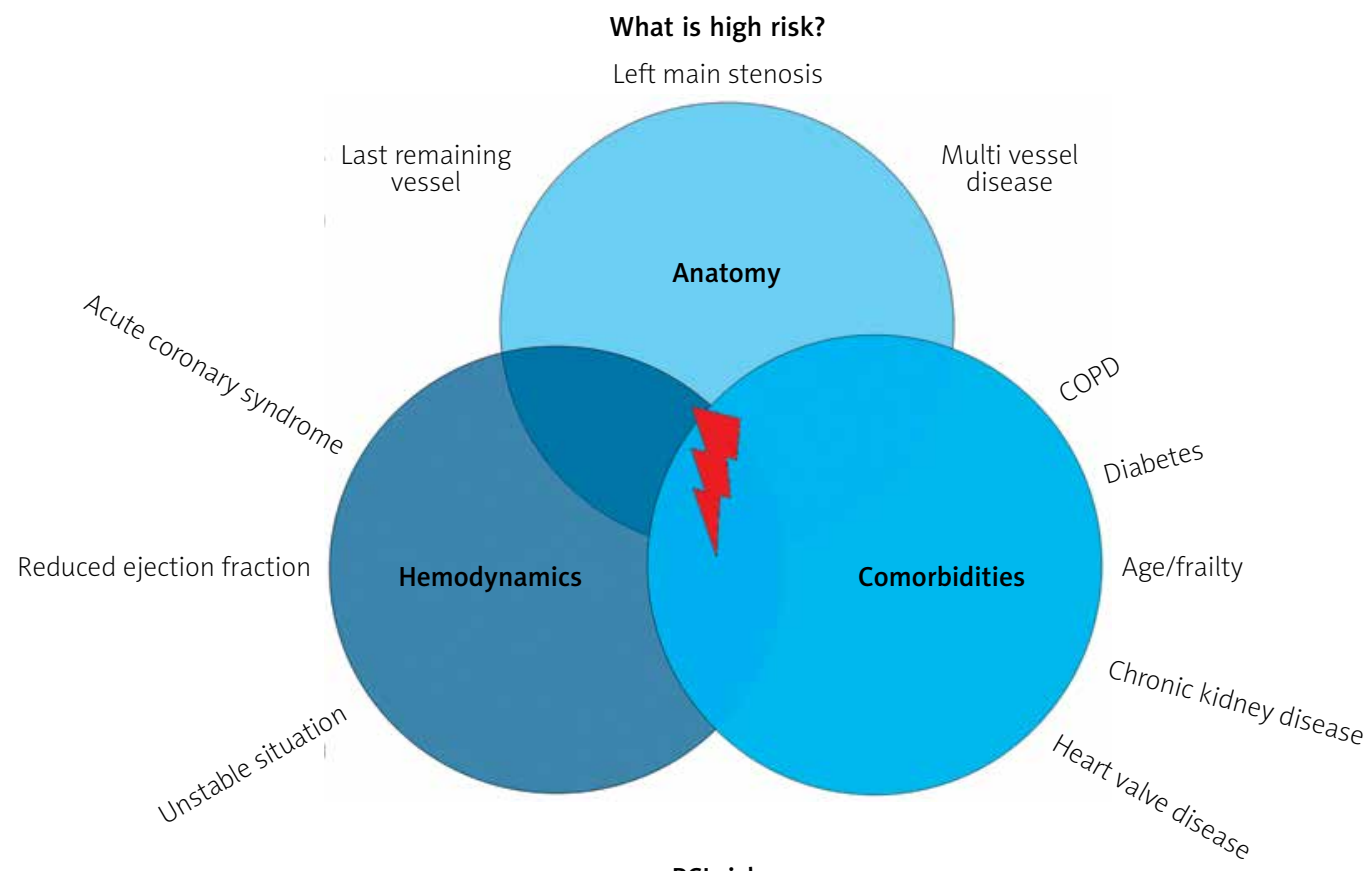

B

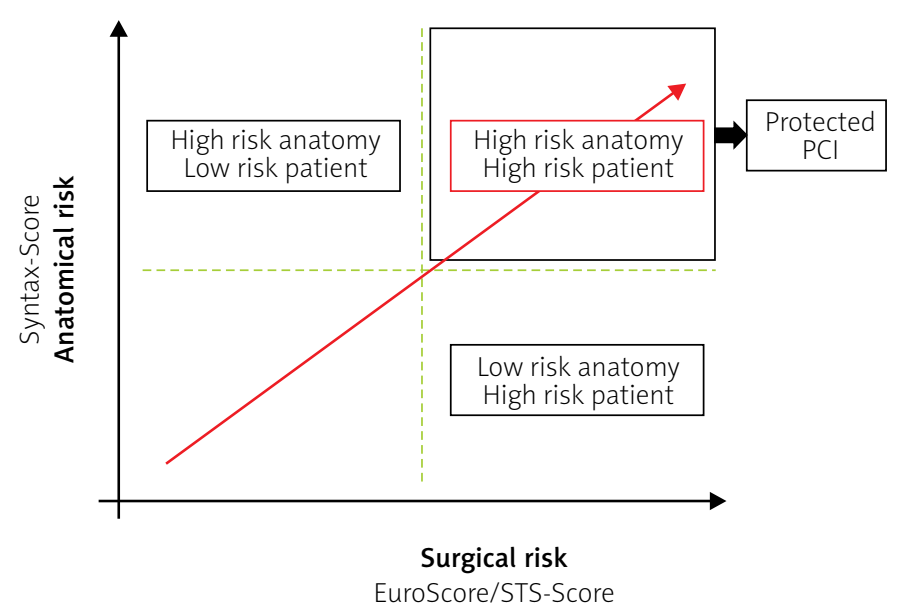

Figure 1. Risk stratification for percutaneous coronary intervention

statistically different between groups, either in the intention-to-treat or in the per-protocol analysis. At the mandatory 90-day follow-up, a strong trend was observed towards a decreased major adverse event rate in Impella 2.5-supported patients compared to IABP-supported patients. This difference was even more pronounced in the per-protocol population $(40.0 \%$ vs. $51.0 \%$ Impella vs. IABP, $p=0.023$ ) [7]. The potential mechanism for this late advantage is supposed to be related to more stable hemodynamic conditions during the procedure, which allowed more complete revascularization including complex techniques such as rotablation [8]. Indeed, further subanalysis revealed that the use of the Impella 2.5 device in patients undergoing more extensive revascularization resulted in fewer periprocedural hypotensive events and was associated with improved 90-day outcomes compared to IABP use.
In the current issue of the journal, Dudek et al. report the initial Polish experiences with hemodynamic support using the Impella CP device in complex, high-risk patients undergoing elective $\mathrm{PCl}$ [3]. And indeed, these were complex, high-risk patients presenting with both high-risk anatomies jeopardizing large amounts of myocardium (i.e. left main and proximal left anterior descending artery stenoses) and with high-risk patient conditions (i.e. age, impaired left ventricular function, previous revascularization, previous myocardial infarction, chronic kidney disease) rendering them at high or prohibitive risk for surgery. The authors are to be congratulated for demonstrating the feasibility and safety of this approach: notably, they did not observe any relevant complications (despite access site hematoma) and no death at 30-day follow-up, although these were their first few cases treated at 6 different centers. 
The high success rate, however, is not surprising and not only based on the fact that the interventions were performed by highly experienced operators at high-volume centers. Without vanity, the authors describe the importance of training, education and preparation (i.e. backup by a vascular surgeon) when engaging and introducing this novel technology. And indeed, there is a learning curve - as in every novel procedure - with substantial room for continuous improvement and refinement in order to further simplify the intervention, but also to broaden the indication. What if the patient had extensive peripheral artery disease precluding transfemoral device insertion? In such cases, a left-axillary approach might be an alternative [9].

In conclusion, complex high-risk $\mathrm{PCl}$ using the Impella CP device for hemodynamic support appears to be a promising way for successful treatment that can be easily introduced into routine clinical practice, as shown by Dudek et al. in this issue of the journal [3].

\section{Conflict of interest}

Philipp Kahlert and Fadi Al-Rashid declare a research cooperation with Abiomed, and Philipp Kahlert has received speaker's honoraria by Abiomed.

\section{References}

1. Serruys PW, Morice MC, Kappetein AP, et al. Percutaneous coronary intervention versus coronary-artery bypass grafting for severe coronary artery disease. N Engl J Med 2009; 360: 961-72.

2. Mohr FW, Morice MC, Kappetein AP, et al. Coronary artery bypass graft surgery versus percutaneous coronary intervention in patients with three-vessel disease and left main coronary disease: 5 -year follow-up of the randomised, clinical SYNTAX trial. Lancet 2013; 381: 629-38.

3. Dudek D, Rakowski T, Sukiennik A, et al. Circulatory support with Impella CP device during high-risk percutaneous coronary interventions: initial experience in Poland. Adv Interv Cardiol 2016; 12: 254-7.

4. Levine GN, Bates ER, Blankenship JC, et al. 2011 ACCF/AHA/SCAI guideline for percutaneous coronary intervention: a report of the American College of Cardiology Foundation/American Heart Association Task Force on Practice Guidelines and the Society for Cardiovascular Angiography and Interventions. Catheter Cardiovasc Interv 2013; 82: E266-355.

5. Amsterdam EA, Wenger NK, Brindis RG, et al. 2014 AHA/ACC guideline for the management of patients with non-ST-elevation acute coronary syndromes: executive summary: a report of the American College of Cardiology/American Heart Association Task Force on Practice Guidelines. Circulation 2014; 130: 2354-94

6. O'Neill WW, Kleiman NS, Moses J, et al. A prospective, randomized clinical trial of hemodynamic support with Impella 2.5 versus intra-aortic balloon pump in patients undergoing high-risk percutaneous coronary intervention: the PROTECT II study. Circulation 2012; 126: 1717-27.

7. Dangas GD, Kini AS, Sharma SK, et al. Impact of hemodynamic support with Impella 2.5 versus intra-aortic balloon pump on prognostically important clinical outcomes in patients under- going high-risk percutaneous coronary intervention (from the PROTECT II randomized trial). Am J Cardiol 2014; 113: 222-8.

8. Cohen MG, Ghatak A, Kleiman NS, et al. Optimizing rotational atherectomy in high-risk percutaneous coronary interventions: insights from the PROTECT II study. Catheter Cardiovasc Interv 2014; 83: 1057-64.

9. Ibrahim AW, Preeyanont P, Rab ST, et al. How should I treat a significant and inoperable left main coronary atherosclerotic disease (LMCAD) in the setting of a severely depressed left ventricular systolic function and severe aorto-iliac atherosclerotic disease. Eurolntervention 2015; 11: 605-8. 